INTERNATIONAL DESIGN CONFERENCE - DESIGN 2018

https://doi.org/10.21278/idc.2018.0125

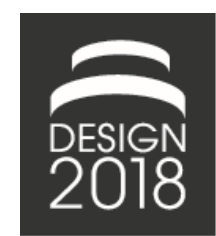

\title{
DATA OBJECTS: DESIGN PRINCIPLES FOR DATA PHYSICALISATION
}

\author{
R. Sosa, V. Gerrard, A. Esparza, R. Torres and R. Napper
}

\begin{abstract}
This paper describes the principles, methods and strategies for the design of everyday objects that embody data - or Data Objects. The work presented in the paper connects the fields of industrial design and data physicalisation to introduce the concept of using data as a design material. To support the creative synthesis of Data Objects the paper provides a literature review, methods and guidance on the creation of Data Objects alongside examples - and possible opportunities, challenges, and future scenarios - for the practice, use and the study of Data Objects.
\end{abstract}

Keywords: big data analysis, design methods, design activities

\section{Introduction}

The term "Data Object" and its acronym DO refer to a type of everyday object configured in the intersection of two domains: data physicalisation and product design. More specifically, DO are usable, functional, and meaningful artefacts whose form and function encode data. These new types of everyday objects range from household devices to street furniture, wearable devices, educational materials, and interactive exhibits. Precedents exist in visual communication, interaction design, and data sculptures, but we suggest that DO remain largely unexplored in product design practice, education, and research. This is evident by the relative absence of product design cases in data physicalisation where most instances consist of volumetric transformations of conventional two-dimensional graphs and maps, sculptural objects whose core character is mainly aesthetic or decorative, or displays of $2 \mathrm{D}$ graphic data representations onto products (Jansen and Dragicevic, 2017).

DO are utilitarian objects encountered in everyday activities for which their data-driven features play a defining role. Whilst these characteristics are not new, we argue that their integration is under-explored in modern product design practice. Therefore, the contributions of this work are to elaborate on the definition of DO, to provide informed means to design DO, and to support their future development. This paper seeks to inspire and inform work around DO with the purpose of establishing ways to support rigorous and creative approaches in the future. The paper begins with a literature review to identify the opportunities for extending the role of design in interacting and making meaning from data. Practical design approaches for DO are then proposed in terms of design principles alongside two design methods with case examples. The paper concludes with a discussion around open challenges and possible areas of relevance including data-driven design activism.

\section{Opportunities for product design in a data-driven world}

The motivation to identify and study the possibilities of DO derives from an awareness that decisionmaking is increasingly driven by - and relies on - data. In the digital age, substantial amounts of data are harvested especially in service of agendas of dominance and hegemony. In a data-driven economy, 
governments and private corporations have access to exhaustive and immediate accounts of the lives of citizens. Key examples that show the potential public benefit of accessing and contributing to large data sets include crowd geo-mapping to predict pandemics (Gregory, 2011), citizen-science (Liu and Singh, 2004), crime prevention with big data (Speer et al., 2017), and predicting risk assessment in child protective services (Pedersen et al., 2004).

The increasing use of 'Big Data' has led to the commodification of data. As a result the collection and use of personal information has become a central political concerns in contemporary society (Jarvis and Daller, 2013). Unresolved questions remain over access, ownership, and transparency, whilst societal action has become crucial to prevent and tackle misuse. Usually justified in terms of safety and efficiency, a trend towards increased restricted access could strengthen in the near future alongside greater data contributions by unwary individuals. As data becomes a more prolific asset to be traded alongside other markers of wealth, product design has the opportunity to address ethical concerns to improve access to data by incorporating it creatively and critically as a design material in professional practice.

The opportunities for creative design action in this data-driven world are vast. Graphic design has long enhanced access to data with early examples including Snow's 1854 map of the cholera epidemic in London affording analysis and understanding of the disease's spread (Crossley et al., 2013). Combined with advances in computation, visual design principles have been applied to make data more understandable and accessible through the use of visual and interactive data representations (Weiner, 2016). The work presented here aims to extend the role of design in interacting and making meaning from data, specifically by escaping "the flatlands of paper and screen" (Tufte, 1990).

Design principles have been used to transfer screen-based interactions to the physical realm through "urban visualisations" (Zamanian and Heydari, 2012). This type of physical representations enables tangible and other sensorial access to data (Jansen et al., 2015). These artefacts embed data in the physical environment, show information in persuasive, objective, and responsible ways, and allow the public to build insights, and potentially engage in participative action (Zamanian and Heydari, 2012).

Traditional societies also collected, analysed and manipulated data in the physical world. The Klikitat and Yakima people kept personal records using an ititamat ("counting the days") to register and recount significant life events on a type of ball of string (Figure 2a) (Schlick, 1994). The Quipu necklace similarly encoded statistical and narrative information in knots (Figure 2b) (de la Cova, 1997). Today, however, fewer data-driven products exist in comparison to other design fields, and where they do exist, they tend to apply rather unsophisticated principles such as surface mapping of two-dimensional graphics (Figure 2c) (Kurbak and Yavuz, 2009).
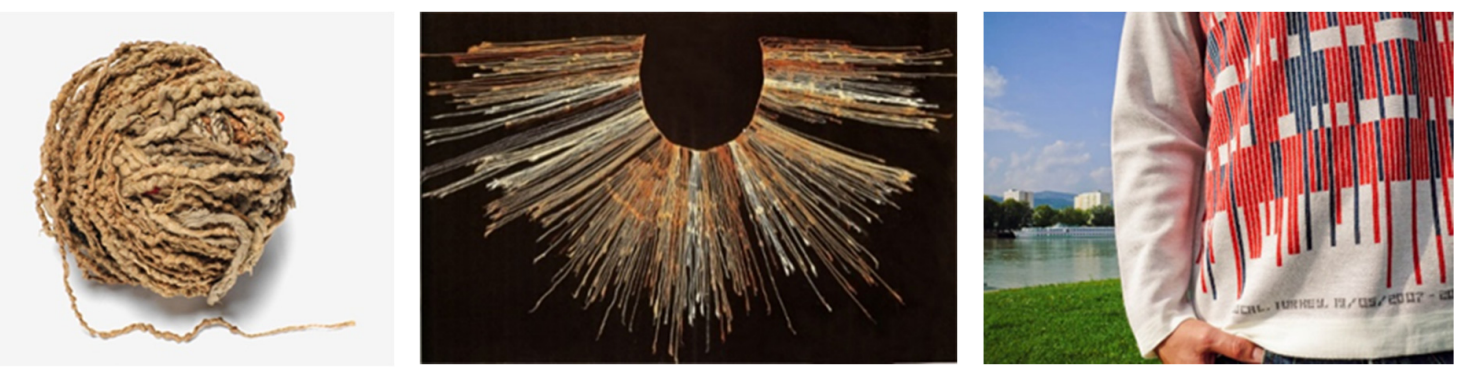

Figure 1. Left: Ititamat ball used as a life-logging device by Native Americans;

Middle: Quipu necklace from the Andes region circa 30th century BC; Right: News Knitter, a garment to visualize customised large-scale data

The field of product design has long espoused the semantic role that designed artefacts play in our daily life (Latour, 1992; Krippendorff, 2006) and yet there has been little use of the extensive quantitative data as a driver of that communicative power. Data has yet to join other design strategies to ethically drive product interaction such as the use of feedback for behaviour change or cues and prompts to incentivise desired and inhibit undesired behaviours (Lockton et al., 2010). Additionally, physical representations in design education are encouraged to 'unlock' alternative cognitive processes and enhance design capabilities (Acuna and Sosa, 2011). Sketching, sculpting, modelling and prototyping 
are all intended to make sense of the materials designers seek to manipulate (Karana et al., 2008). The same justifications could be applied to the processes required to shape data as a design material.

This paper sets out to describe how product design principles might inform design with data. In addition to the creative opportunities available to further product design practice through data physicalisation, we identify an ethical responsibility to design for access to data and contribute to the critical engagement with 'Big Data'.

\section{Designing Data Objects}

In this section, principles and strategies for the design of Data Objects are described. Building on the framework developed by Vande Moere and Purchase (2011) to examine the role of design in information visualisation, we draw from a wide literature to inform the specific practice of information visualisation in product design.

\subsection{Design principles}

We identify four singular design principles to orient design activity towards the differentiating characteristics of DO. These principles focus specifically on the data considerations of DO and are intended for use alongside existing design principles (Martin and Hanington, 2012), and data visualisation principles (Tufte, 1990, 2001; Kostelnick, 2008).

DO Principle \#1: Treat data as a new type of material to design with.

Just like design-led evaluation and selection of materials and transformation processes (Ashby and Johnson, 2010), a design-led treatment of data seeks to support an informed, responsible, and organised way to transform raw or statistically processed data into Data Objects. In the process of making tangible the intangible, selection criteria for data must be considered in relation to the affordances provided. Conceived as a new type of material, data have technical characteristics (quantifiable) to be mapped onto physical artefacts, i.e., scale and statistical properties including groupings and distributions. Data also have intangible characteristics which need to be identified and integrated in the design of DO to define the design intentions and directions shaped by the data. In other words, DO that encode data about income, time-use, or human trafficking deal with very different units and their design needs to consider both the direct and the "second and third-order characteristics" (Karana et al., 2008) of the cultural, contextual, and human dimensions of the data.

DO Principle \#2: Design for access and (re)interpretation of the data embedded in the object.

With access to data at their core, DO rely on the identification of a source data set to be represented through an easily recognised object form or function - with units, scales, relations, types, and quality of the data carefully selected for perceptibility and coherence (Norman, 2013; Jansen et al., 2015). Complex data sets can be introduced through layered multisensory interactions which support users to interpret the data in manageable parts (Jansen et al., 2015). Principles of organisation - including framing, mapping, chunking, hierarchies, layering, priming, similarity, and comparison are applicable, while iconic, indexical, and symbolic signifiers to support data recognition and exploration (Chandler, 2001). These symbols can be used as 'wayfinding' elements to reduce formal instruction and prevent errors in data interpretation.

Ultimately, the codification of a DO is determined by a user's existing cultural conventions and values - including their learned expectations, behaviours, beliefs and interpretations associated with data (Weinschenk, 2011). The selected data set, its presentation, interface and use of symbols must be understood in relation to these conventions. Essentially, designers of DO must ensure that the data embedded in the artefact can be easily accessed and re-codified by users (Freire, 2000). Data must be truthfully represented for accurate access without requiring additional explanations (Tufte, 1990). Designers of DO need to anticipate people asking a range of "Why?" questions when interacting with DO. Rather than DO designed to transmit a sterile message to end the conversation, they are better suited to support a multiplicity of ideas to trigger conversations framed by intuitive access to concrete and specific data. As a result, DO are not about the designers' views (except incidentally), and their focus is on giving agency to users. To this end, testing iteratively during the design process is essential to assess 
their capacity to spark many and genuine questions; their power as tools for thinking and engaging with topics and new understandings rather than as soapboxing or propaganda; and for legibility, i.e., do people react and interact with the DO based on the right data?

DO Principle \#3: Design for cognitive and emotional engagement with the data embedded in the object.

DO are more successful when they afford interaction and use in multiple levels of engagement: from first impressions as seemingly innocuous everyday objects to further use and interactions as the data embedded in the object, its function, and its use are revealed. DO are likely to fully transmit the data they carry when in repeated use or close contact, including active and passive interaction modes (Marcus, 2016). Emotional and cognitive connections to the data emerge through playful, explorative, or purposeful interactions with the object (Jordan, 2003). Conditioning methods which associate an interaction with an unconscious physical or emotional response can orient users to interpret the data. Interactions can be designed with an acknowledgement of user progress in knowledge and skill through object use by providing feedback that sustains engagement. Principles of motivation, rewards, flows, information seeking, progress-mastery-control, competition, and user autonomy can be helpful in this regard (Weinschenk, 2011). Where there are multiple users, this type of feedback can be used alongside mechanisms of social cognition such as homophily; imitation and empathy; collaboration; peer pressure and compliance; and humour to encourage dialogue between users (Freire, 2000; Datta and Mullainathan, 2014).

At a basic level, designing DO for interpretation and engagement draws from principles of communication design. In both areas, what users do with the information or the data is what is important. In the same way as communication design elements afford ways to represent and transform hitherto two-dimensional information for example using typography or animation techniques on a time axis, the physical properties of DO affords designers new ways to manipulate data on three physical axes as well as a fourth (time) axis. Other elements such as materials, texture, colour, and form can also be explored as representational opportunities to shape data. Ultimately, sustained user engagement with the DO will be determined by the relevance of the data presented to the user - defined by its ability to shock, inform, surprise and delight. The selected data, type of interaction, and opportunities for decoding and interpretation must resonate with the intended users, and is likely to emerge from iterative prototyping or co-design activity (Manzini and Coad, 2015).

DO Principle \#4: Design to empower people to use the data to rethink and challenge the status quo.

By definition, DO are factual but can hardly be considered neutral, i.e., they accurately map quantitative information, but its selection, organisation, and representation is purposeful. DO are peculiarly apt to create a counter-narrative - defying unquestioned stereotypes and tropes dictated from the top- by inviting users to work with data. Whilst prosaic versions of DO can be propagandistic, the ultimate aim for DO is to set the stage, frame the dialogue, and highlight issues and tensions in ways which open possibilities and create opportunities for inclusive discussion (DiSalvo, 2012; Sosa et al., 2016). Physically embedding mnemonic devices, imagery, analogies, and archetypes within the data object provide mechanisms through which people can pose new types of questions. This is supported by offloading strategies using the physicality of DO to alter the information processing requirements and reduce cognitive demand (Nielsen and Mack, 1994; Weinschenk, 2011). Techniques which ground existing mental models in a real and manageable experience prompt reactions that would not otherwise have been elicited. Data can be embedded in ways that enhance the lived experience and demand immediate action. Embedding incentives for preferred behaviours or perceptions without restricting options or significantly changing incentives/penalties can nudge users towards conclusions and their own generative questions (Berger, 2014).

Tentative encodings of data in DO should inspire and invite collective interpretation and dialogue rather than prescribe themes and interpretations from a preordained perspective (DiSalvo, 2012; Gerrard and Sosa, 2014). Projecting possible future scenarios associated with a present situation, or by tracing the histories, discourses, underlying issues, causes, and structures of a present situation can foster dialogue between perspectives and create new publics (DiSalvo, 2009; Dunne and Raby, 2013). Ultimately, possible behaviour change will be determined by which schemata and frameworks users exist within. The data selected for critique, the manner in which the critique is presented and the incentives to critique 
must be formulated with an understanding of these schemata in order to make abductive inferences and assumptions about how information will be processed and questioned. When relevant, design decisions for DO would ideally include their larger context, the immediate setting, the publics, and their interactions with and through the built environment (Latour, 1992).

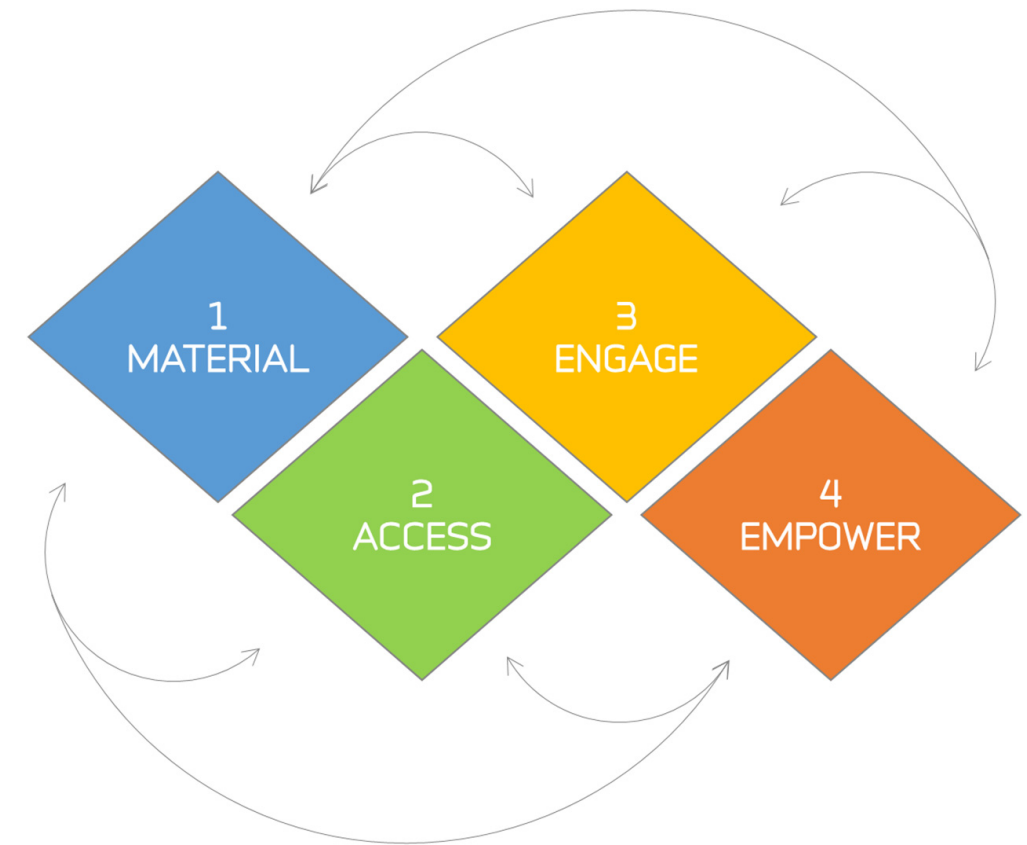

Figure 2. Four design principles for DO: Material, access, engage, empower

An example is described following the DO principles. "Trashball" by Irwan Ahmett and (Latour, 1992). Trashball is a playful object intended to draw attention to the amount of trash in low income areas of Jakarta, Indonesia. Trash is collected and gradually formed into a ball which is used to play games of catch, football, human skittles, and eventually - as the ball becomes very large - rolled (paraded) around the city. The materiality of the object is directly associated with the trash incorporated as the ball is built in subsequent layers of flat material -first DO principle. In this case the DO is simultaneously about and made of an issue which is problematic because of its pervasive physical presence: urban waste such as discarded packaging. The object engages the public by inviting them to help build the ball, a strategy particularly successful with the youth -second DO principle. Because Trashball prioritises situated and collaborative making, issues of scale are also mapped directly onto an object which strengthens its message by continuously growing in size thus mapping the magnitude of the problem -third DO principle. A visual and tangible record of the amount of trash is formed along its route, also defined by those playing and interacting with the ball. The ball grows satisfyingly quickly providing sufficient motivation and feedback for users to continue interacting with it, and frequent breaks in building to experiment with new games with the ball provide additional engagement -third DO principle. As the ball grows to an absurd size for traditional ball games and becomes more difficult to control - sometimes even dangerous - the urgency of the environmental message becomes clear and unavoidable. Those involved in creating the ball - potentially also partly responsible for the trash that it is made from become advocates of the message as they are physically and emotionally connected to the ball, and justify its existence as a symbol of poor waste management practices -fourth DO principle.

\section{Design methods for Data Objects}

We consider here two methods for the design of Data Objects: "Data to Object" and "Object to Data". In the former, the source data guide the design process. In the latter, the target object guides the design process. The first method is more "open-ended" as it does not prescribe the type of output other than 
being a physical object. The second method is a more directed approach, as it seeks to re-imagine a specific type of object.

\subsection{Method I: "Data to Object"}

One way to structure the design process of DO is to take the materiality of the data as the starting point, and proceed to shape the selected data into an everyday object. This DO method is called "Data to Object" and consists of the following steps:

1. Select data sources, filter them, and examine the material properties of the data as you list as many conceivable ideas as possible to orient the design of DO based on the data: the publics, the messages, the goals, the scenarios.

2. Build initial 2D and 3D representations of the data to get a sense of amounts, volumes, scales, proportions, use of colours, shapes. Develop a feeling of the physical properties and the sensorial affordances conveyed by the data.

3. Develop concepts for objects related to the dataset. Decide on the messages to be encoded in the data object and consider these in terms of the perceptual affordances in the data. Develop concepts into physical and social dimensions. Features derived from the data can distort the object - break expected symmetry, proportion, or scale - while metaphors and symbols can be used to transmit meaning. Consider how the geometry can change product function and a user's expected interaction with the object.

4. Prototype and evaluate the power of the data object to convey data when in use. As with any design, it is crucial to involve target users in the evaluation of the data object through prototyping and possibly by inviting them to select, curate, and work with the data. Through physically engagement, data objects seek to share, build dialogue, and build interpretations. Be prepared to go back to prior steps to re-examine previous decisions and consider other alternatives.

\subsection{Method II: "Object to Data"}

A second way to structure the design process of DO is to take the materiality of a type of everyday object as the starting point, and proceed to shape an instance object based on the features of the data selected. This DO method is called "Object to Data" and consists of the following steps:

1. Select the type of objects to be redesigned or re-interpreted and examine their material properties including function, shape, semantics and, historic and cultural relevance.

2. Develop data concepts related to the object. Explore data opportunities which relate to the object semantics. Speculative objects may be considered, i.e., versions that are impractical or defy common-sense. Different use scenarios for the object might also be considered, along with different users and modes and scenarios of use.

3. Play with the perceptual and conceptual mappings between data and object by manipulating 2D and 3D representations. Develop the connections between physical features, data, and the possible decoding alternatives available to users. Features derived from the data can be used to distort the object, for example to break the expected symmetry, proportion, or scale to map the data onto the object. Building physical or virtual is the main activity at this stage.

4. Prototype and evaluate the ability of the data object to convey data when in use. As with any design, it is important to involve target users in the evaluation of the data object through prototyping and possibly inviting them to participate in the process. Through physical engagement, data objects seek to share, build dialogue, and build interpretations. Be prepared to go back to prior steps to re-examine previous decisions and consider other alternatives.

Due to space constraints, we focus next on the analysis of a case study that illustrates the second method "Object to Data" since this has been less obvious for those approximating the design of DO for the first time. The examples originate from recent practice facilitated by the authors. Based on this experience, we identify three starting points for DO design briefs transitioning from more bounded to more openended. In settings where more focus is required, define and provide to participants a source data base (Method I) and/or a target type of object (Method II). When a balance between freedom and similarity 
of outcomes are required, such as in competition or studio projects with common rubric criteria, participants can be asked to select and curate data from a subset provided (Method I), and/or they can select from candidate object types or a set of building materials supplied. Thirdly, when more freedom is desired, a theme or overall goal for the project can be set, giving agency to participants to select the dataset (Method I) or to define the target objects of their own interest (Method II) -and their own choice of materials. Most likely, a combination of these strategies will be appropriate, considering the characteristics of the project, the participants, the desired outcomes, i.e., a museum exhibition, a school project, a public co-design workshop, etc.

\section{Case study}

This section presents a case of study of products designed by the first and second authors of this paper from 2007 to 2017 as part of their design practice. These projects respond to the design brief of designing data objects by applying Method II as described in the previous section. The dual function of a "table" is playfully intertwined in these projects to create data tables both as "a set of facts or figures systematically displayed, especially in columns" and "a piece of furniture with a flat top and one or more legs, providing a level surface". The everyday tables designed in these projects aimed to enable users to physically experience and explore the meanings and consequences of the data sets selected.

\subsection{Income Tables}

The Income Tables presented in Figure 3 in a CAD model represent quartiles of annual income per capita in Mexico (green table) and New Zealand (blue table) for the year 2007. In this example, the difference between the volumes of the table legs represents economic disparity. In one of the tables, the disparity becomes so large that the object becomes awkward with little legroom available and a prominent disproportion. The intention to disrupt the geometry of these objects is to promote discussion of those sitting at the table about the dysfunction of economic disparity, which can become precarious or unsafe after an extreme degree of disproportion and imbalance.

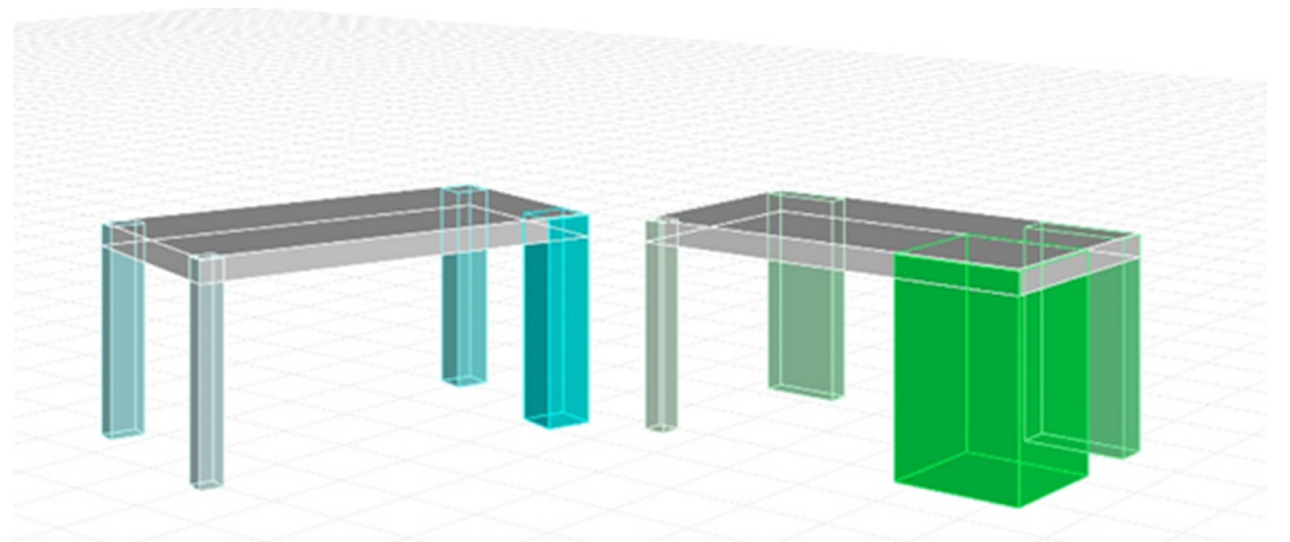

Figure 3. Income Tables (2007 by first author); leg volume represents quartiles of annual income per capita

In this case, the focus of the design became the four legs of a canonical table, which were directly used to map the data structured in quartiles -first DO principle. The main purpose of this design was to serve as an entry example for Data Objects workshops and projects, therefore the object design was kept as simple as possible for participants with limited design literacy or experience to visualise an example DO. To this end, a simple top slab of constant dimensions is left unmodified between the tables and no additional elements or decorations are incorporated. In this sense, the mapping between table legs and income quartiles is easily established -second DO principle. From that point, we have observed that it becomes easy for people to start thinking of their own data of interest that they can imagine meaningfully 
divided in fourths and mapped onto a table's legs -third DO principle. In testing scenarios, audiences have made explicit the notion that the physical presence of these tables and their constant shaping of the interactions around them, carry a powerful message in ways that are not possible even with advanced visualisations on a screen, which can be effective but whose message dwindles as the visualisation disappears from the screen or the screen is shut down. A wide range of variants of these tables have been created in class activities and design workshops mapping imbalances and inequality along a variety of topics and populations.

\subsection{This Table is Occupied}

The Occupy Movement of 2012 was concerned with highlighting inequities in global wealth distribution. "This Table is Occupied" is a play on the data voiced by the protesters to show that the richest population $20 \%$ owns $82.7 \%$ of global wealth while the remaining $80 \%$ share only $17.3 \%$, with the poorest $40 \%$ owning virtually nothing. The data is represented in a DO table which seats 5 people - each representing $20 \%$ of the global population. One member of the group has access to $82.7 \%$ of table surface area while the remaining five people have access to $17.3 \%$ - and, in reality, two of those five have very little access to any part of the table surface. Not only does the person representing the wealthiest $20 \%$ have access to the most table space, they are separated from the majority - with a large barrier between them and the other 4 users, and facing a different direction. This divide is a reflection on reality, where the world's richest are increasingly separated from 'the rest' by systems which provide few opportunities for dialogue between income groups. A secret suggestion for action is embedded in the table design where if the table is flipped over, the distribution of table surface is the same for each person. The difficult activity of flipping a concrete table represents the difficult task of flipping current economic systems which are needed to overcome disparities in global wealth distribution.
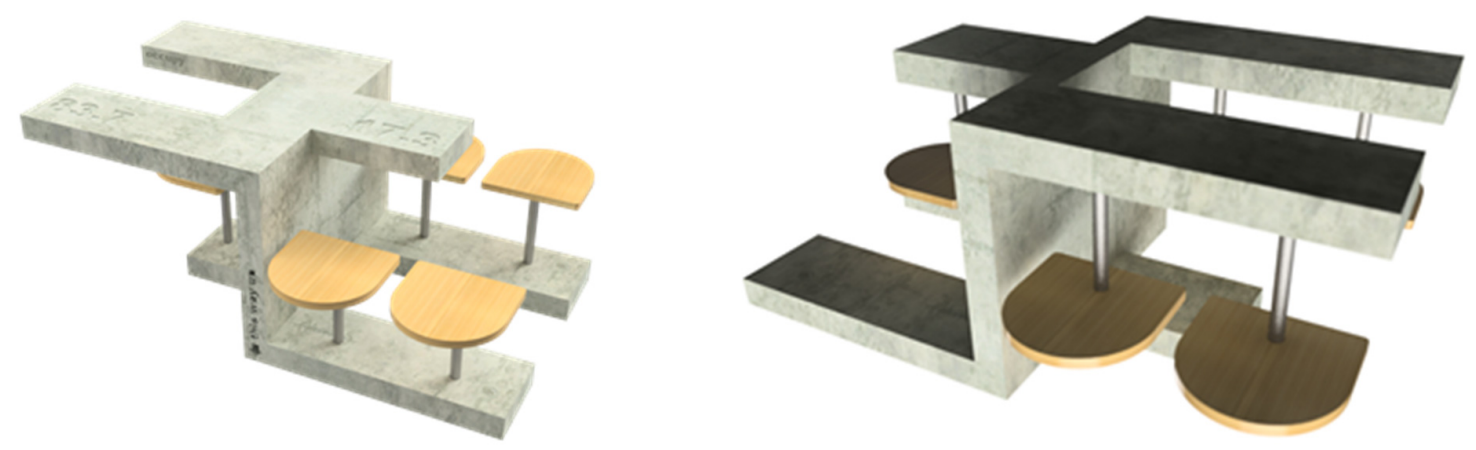

Figure 4. This Table is Occupied (2017 by second author)

In this case, the focus of the design was the surface of the table, which was directly used to map the data as a percentage of surface area -first DO principle. The original data set was expressed as quintiles but a simpler form of separating the extreme quintile from the remaining four was decided to maintain clarity and the greatest data outlier to be recognised. The main purpose of this design was to serve as an example of the use of layering to assist users to decode the form of the table through use. The seats are left unmodified and invite the user to experience the data through available table surface through first hand interaction -third DO principle. In use, the physical form of the table shapes the interactions between people, communicating a visceral and immediate experience of hidden social realities. The need to 'flip' the table to enable a discussion between all users prompts a more solution driven conversation about taking collaborative and empowered action -fourth DO principle.

Whilst users can interact with these tables without knowing what they represent, their physical presence provides a subtle but ever pervasive reminder of things that "aren't right" and which call for our attention. Crucially, the tables represent the hard facts, around which people can share their views and learn from each other in conversations such as those afforded by formal round-table discussions or by more casual café settings. The experience of sitting at these tables may be more likely to evoke empathy 
in those engaged in a provocative and heated exchange of ideas, as they are placed in a facsimile of the situation being described. Moreover, 'non-table' versions of these examples are also frequently created in design workshops, for example the data for the Income table can be mapped onto a range of artefacts with four subcomponents such as a cutlery set where the spoons represent the first quartile, forks the second, knifes the third, and the tea spoons the fourth -the oversized spoon would align well with a metaphor of income used for subsistence. Other object-types besides tables are also suitable for the design method "Object to Data". For example, in a recent workshop, the design of "Altares de Muertos" traditionally used in Day of the Death celebrations in Mexico has been used to map a range of crimes such as those against women, migrants, and casualties from gun violence and war.

\section{Design Objects for Design Activism (DOxDA)}

Design-led activism is defined as design applied "to create a counter-narrative aimed at generating and balancing positive social, institutional, environmental and/or economic change" (Fuad-Luke, 2013). Thorpe's (Thorpe, 2012) criteria of design as activism include: to publicly reveal or frame a problem or challenging issue; to make a contentious call for change; to work on behalf of a neglected, excluded or disadvantaged group; and to disrupt routine practices, or systems of authority. DO are particularly suited for design activism through the enabling of people to access and make sense of information which is useful to enhance society. Data Objects for Design Activism, or DOxDA transcends working on behalf of others. Instead, DOxDA aim to re-frame key features of the present reality and expose people to significant issues through verifiable and accurate data. This gives DO an advantage as part of the design toolbox for creative activism whilst rejecting the self-importance of design as a "major role in triggering and supporting social change" (Manzini and Coad, 2015). Instead, designers creating DO seek to provide a verifiable backdrop where conversations and dialogue led by the public can be grounded and articulated.

In this vein, DO are considered as non-human agents, i.e., "material presenters [that] support participation" (Bjögvinsson et al., 2012). The key notion in this agency is access; physicalisations aim to substantially increase the impact of making information comprehensible to wide groups of people. This can be portrayed as a progression from raw data inaccessible to human understanding, proceeding to highly specialised statistical means exclusive for data analysts, to info-visualisations aimed at a general audience, yet still demanding sophisticated visual literacy skills and specialised technical resources such as displays and online access. DO seek to build opportunities for people to intuitively reason about data through daily interactions with the built environment, with the goal to support wellinformed dialogue about the present.

DOxDA can be considered as leverage points (Meadows, 1997) where a small "shift in one thing can produce big changes in everything". DO are aimed at changing "information flows" by affecting the physical structure of daily life. DO seek to provide feedback to address the "tendency on the part of humans to avoid accountability for their own decisions" (Meadows, 1997). Moreover, DO aim to make the goals of the system clearer since "people within systems don't often recognise what whole-system goal they are serving" (Meadows, 1997). At its best, DO are built to trigger mind-set or paradigm shifts by making information (physically, socially) present in everyday life, thus making explicit the "deepest set of beliefs about how the system works" and "pointing at the anomalies and failures of the old paradigm" (Meadows, 1997).

DOxDA relate to other practices, including Speculative Design, but their orientation is on the present, or rather, on alternate versions of the present. In this sense, DOxDA are more reflexive than speculative, as they are not focused on presenting what the future could be, but supporting the construction of what the present could mean for different actors. By questioning the present trajectories of key well-being indicators, DOxDA can inform future-oriented activism fields. Figure 5 shows the distinction between speculative approaches and DOxDA: whilst the former as shown in the left represents the present as a single point (Dunne and Raby, 2013), the design of DOxDA avoids such assumption by recognising the need to account for probable, plausible, and possible versions of the present, and to contribute with data representations that help different agents make inferences about the current state of the system, therefore by logic widening the spectrum of probable and plausible imagined futures. 


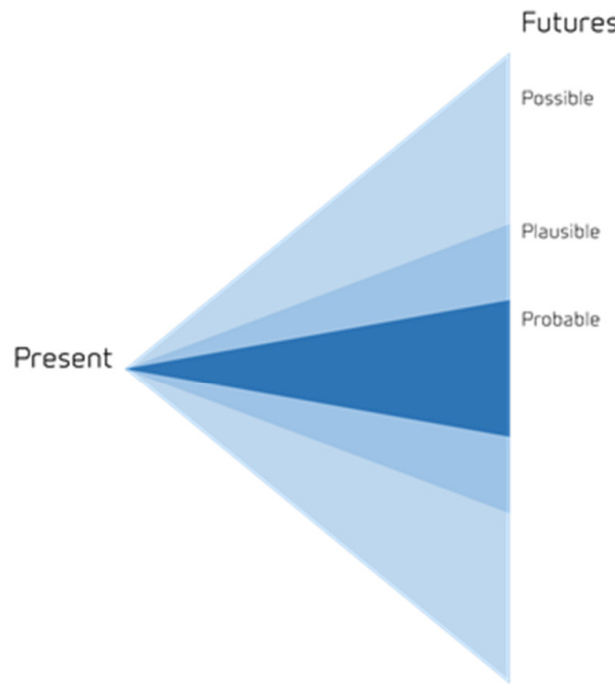

Speculative Design

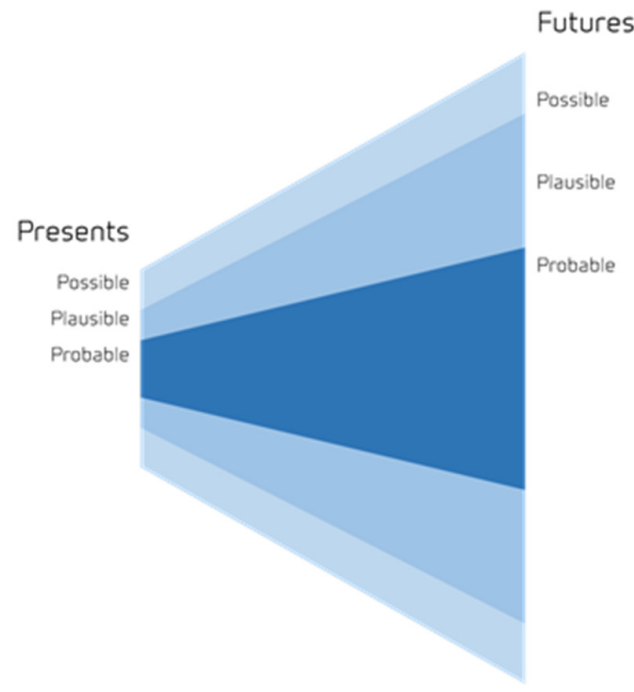

Data Objects for Design Activism (DOxDA)

Figure 5. Data Objects as instruments to understand the probable, plausible, and possible versions of the present, leading to an expansion of possible futures

An alternative option for DOxDA to address in the future is the encoding of speculative data, i.e., data that represents fictitious futures and desirable realities. This could be done as a way to compare present realities with idealised models (utopian), with future projections of current trends (dystopian), or with the anticipated effects of intervening in the system. Visualising alternate versions of the present is important because social movements, interest groups and activists tend to embrace a shared set of beliefs or perceptions. The increasing complexity of social and environmental changes makes it difficult for people to perceive, and difficult to engage with.

\section{Discussion}

The design of DO merges three important design dimensions: product semantics, usability, and product function. The motivation to connect these three product characteristics is a response to a social evolution towards an increasingly data driven world where access to - and understanding of - data has become an essential human capability. The wider purpose of info-visualisation is to "help people think about, explore, and share data" (Jansen et al., 2015). The intention to embed data in designed products is an attempt to provide an alternative medium through which to democratise data - expanding these intentions to the physical world.

While objects have always had the ability to communicate, it has been the normal purview of the product designer for the product to communicate internal attributes. These internal attributes are the classical elements of industrial design, for example the form language of a car communicating dynamism, or an armchair comfort. This form of communication assists the user in making informed decisions about using these products, and indeed is manipulated by the industrial designer to achieve particular ends some as altruistic as product purpose, and others which may be viewed nefariously such as simply selling more stuff. What DOs enact is an ability for products to communicate information beyond their own realm. This may be viewed as a convergence of industrial and communication design, or alternatively, a convergence of ordinary products with the "status object", intended to communicate projected values of the owner. It also opens an opportunity for more focussed agency or activism on the part of the objects, and their designers.

\section{References}

Acuna, A. and Sosa, R. (2011), "The complementary role of representations in design creativity: sketches and models", In: Taura, T. and Nagai, Y. (Eds.), Design Creativity 2010, Springer London, London. https://doi.org/10.1007/978-0-85729-224-7_34 
Ashby, M.F. and Johnson, K. (2010), Materials and Design: the Art and Science of Material Selection in Product Design, Butterworth-Heinemann, Oxford.

Berger, W. (2014), A More Beautiful Question: The Power of Inquiry to Spark Breakthrough Ideas, Bloomsbury Publishing, Chicago, USA.

Chandler, D. (2001), Semiotics: The Basics, Routledge, New York.

Crossley, S., Salsbury, T. and McNamara, D.S. (2013), "Validating lexical measures using human scores of lexical proficiency", In: Jarvis, S. and Daller, M. (Eds.), Vocabulary Knowledge: Human ratings and automated measures, John Benjamins Publishing Company. https:/doi.org/10.1075/sibil.47.06ch4

Datta, S. and Mullainathan, S. (2014), "Behavioral Design: A New Approach to Development Policy", Review of Income \& Wealth, Vol. 60 No. 1, pp. 7-35. https://doi.org/10.1111/roiw.12093

de la Cova, A.R. (1997). Incas Quipu. [online] Available at: http://www.latinamericanstudies.org/quipu.htm (accessed 11/2017).

DiSalvo, C. (2009), "Design and the Construction of Publics", Design Issues, Vol. 25 No. 1, pp. 48-63. https://doi.org/10.1162/desi.2009.25.1.48

DiSalvo, C. (2012), Adversarial Design, MIT Press, Cambridge, Massachusetts.

Dunne, A. and Raby, F. (2013), Speculative Everything: Design, Fiction, and Social Dreaming, The MIT Press, Cambridge, Massachusetts.

Freire, P. (2000), Pedagogy of the Oppressed, Continuum, New York.

Gerrard, V. and Sosa, R. (2014), "Examining participation", Participatory Design Conference, Association for Computing Machinery, pp. 111-120. https://doi.org/10.1145/2661435.2661451

Gregory, R.J. (2011), "Validity and test development", In: Gregory, R.J. (Ed.), Psychological Testing: History, Principles, and Applications, Global Edition, Pearson Higher Ed, New York.

Jansen, Y. and Dragicevic, P. (2017), List of Physical Visualizations and Related Artifacts. [online] Available at: http://dataphys.org/list/ (accessed 11/2017)

Jansen, Y., Dragicevic, P., Isenberg, P., Alexander, J., Karnik et al. (2015), “Opportunities and Challenges for Data Physicalization", 33rd Annual ACM Conference on Human Factors in Computing Systems, Seoul, Republic of Korea, ACM. https://doi.org/10.1145/2702123.2702180

Jarvis, S. and Daller, M. (2013), Vocabulary Knowledge: Human Ratings and Automated Measures, John Benjamins Publishing. https://doi.org/10.1075/sibil.47

Jordan, P.W. (2003), Designing Pleasurable Products: An Introduction to the New Human Factors, Taylor \& Francis, London.

Karana, E., Hekkert, P. and Kandachar, P. (2008), "Material considerations in product design: A survey on crucial material aspects used by product designers", Materials and Design, Vol. 29 No. 6, pp. 1081-1089. https://doi.org/10.1016/j.matdes.2007.06.002

Kostelnick, C. (2008), "Beyond the Tyranny of the Pixel: Exploring the Physicality of Information Visualization", IEEE Transactions on Professional Communication, Vol. 51. https://doi.org/10.1109/IV.2008.84

Krippendorff, K. (2006), The Semantic Turn: A new Foundation for Design, CRC/Taylor \& Francis, Boca Raton. https://doi.org/10.4324/9780203299951

Kurbak, E. and Yavuz, M.M. (2009), NewsKnitter. [online] Casualdata. Available at: http://casualdata.com/newsknitter

Latour, B. (1992), "Where are the missing masses? A sociology of a few mundane artefacts", In: Bijker, W. and Law, J. (Eds.), Shaping Technology-Building Society. Studies in Sociotechnical Change, MIT Press, Cambridge Massachusetts.

Liu, H. and Singh, P. (2004), "ConceptNet — a practical commonsense reasoning tool-kit", BT Technology Journal, Vol. 22 No. 4, pp. 211-226. https://doi.org/10.1023/B:BTTJ.0000047600.45421.6d

Lockton, D., Harrison, D. and Stanton, N.A. (2010), “The Design with Intent Method: A design tool for influencing user behaviour", Applied Ergonomics, Vol. 41 No. 3, pp. 382-392. https://doi.org/10.1016/j.apergo.2009.09.001

Manzini, E. and Coad, R. (2015), Design, When Everybody Designs: An Introduction to Design for Social Innovation, MIT Press, Cambridge, Massachusetts.

Marcus, A. (2016), Design, user experience, and usability: design thinking and methods, Springer, Switzerland. https://doi.org/10.1007/978-3-319-40409-7

Martin, B. and Hanington, B.M. (2012), Universal Methods of Design: 100 Ways to Research Complex Problems, develop innovative ideas, and design effective solutions, Rockport Publishers, Beverly, MA.

Nielsen, J. and Mack, R.L. (1994), Usability Inspection Methods, Wiley, New York. https://doi.org/10.1145/259963.260531

Norman, D.A. (2013), The design of everyday things, Basic Books, New York.

Pedersen, T., Patwardhan, S. and Michelizzi, J. (2004), "WordNet: Similarity: measuring the relatedness of concepts", Proceedings of the 19th National Conference on Artifical Intelligence, AAAI Press. 
Schlick, M.D. (1994), Columbia River Basketry: Gift of the Ancestors, Gift of the Earth, University of Washington Press, Seattle, Washington.

Sosa, R., Torres, R., Cervera, F. and O, R. (2016), "Eliciting creative views of Tertiary Education through speculative future scenarios: Robot "O"', TERNZ Conference Tertiary Education Research in New Zealand, Dunedin.

Speer, R., Chin, J. and Havasi, C. (2017), "ConceptNet 5.5: An Open Multilingual Graph of General Knowledge", Proceedings of the Thirty-First AAAI Conference on Artificial Intelligence (AAAI-17), pp. 4444-4451.

Thorpe, A. (2012), Architecture and design versus consumerism: how design activism confronts growth, Earthscan, New York.

Tufte, E.R. (1990), Envisioning Information, Cheshire, Graphics Press, Connecticut.

Tufte, E.R. (2001), The Visual Display of Quantitative Information, Graphics Press, Cheshire, Connecticut.

Vande Moere, A. and Purchase, H. (2011), "On the role of design in information visualization", Information Visualization, Vol. 10 No. 4, pp. 356-371. https://doi.org/10.1177/1473871611415996

Weiner, E. (2016), The Geography of Genius: A Search for the World's Most Creative Places from Ancient Athens to Silicon Valley, Simon and Schuster, New York.

Weinschenk, S. (2011), 100 Things Every Designer Needs to Know about People, New Riders, Berkeley, CA.

Zamanian, M. and Heydari, P. (2012), "Readability of texts: State of the art", Theory and Practice in Language Studies, Vol. 2 No. 1, pp. 43-53. https://doi.org/10.4304/tpls.2.1.43-53

Dr. Ricardo Sosa

Auckland University of Technology, Design and Creative Technologies

Governor Fitzroy Place, 1010 Auckland, New Zealand

Email: ricardo.sosa@aut.ac.nz 